\title{
Behavioural Genetics in the Postgenomics Era
}

Evan Charney, Duke University, Durham, North Carolina, USA

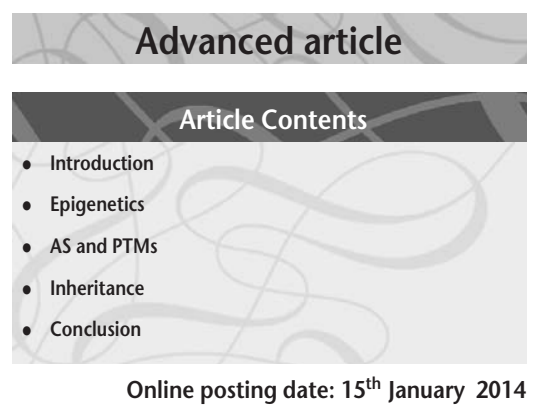

There is growing evidence that the complexity of higher organisms does not correlate with the 'complexity' of the genome (the human genome contains fewer protein coding genes than corn, and many genes are preserved across species). Rather, complexity is associated with the complexity of the pathways and processes whereby the cell utilises the deoxyribonucleic acid molecule, and much else, in the process of phenotype formation. These processes include the activity of the epigenome, noncoding ribonucleic acids, alternative splicing and post-translational modifications. Not accidentally, all of these processes appear to be of particular importance for the human brain, the most complex organ in nature. Because these processes can be highly environmentally reactive, they are a key to understanding behavioural plasticity and highlight the importance of the developmental process in explaining behavioural outcomes.

\section{Introduction}

The completion of the Human Genome Project has not lived up to the expectation regarding the identification of causal, risk factor or predisposing alleles for human behaviour, both normal and abnormal. There have been some successes, for example, a polymorphism of the e4 version of the APOE gene increases an individual's risk for developing late-onset of Alzheimer disease. But instances such as this are an exception. To date, not a single riskfactor allele has been identified for either the most prevalent mental illnesses (schizophrenia, autism, bipolar disorder and depression) or behavioural variation within a 'normal' range (e.g. intelligence and personality) (Turkheimer, 2012). From a postgenomics perspective, this

eLS subject area: Genetics \& Disease

How to cite:

Charney, Evan (January 2014) Behavioural Genetics in the

Postgenomics Era. In: eLS. John Wiley \& Sons, Ltd: Chichester. DOI: $10.1002 / 9780470015902 . a 0025250$ is not at all surprising, particularly with regard to behavioural variation associated with the healthy brain.

One of the surprising findings of the Genome Project was that the human genome contains an estimated 20000 protein coding genes, less than maize (i.e. corn), which contains over 32000 protein-coding genes, and close in number to the nematode, with approximately 19000 . And many genes appear to be preserved across species. Surely, the distinctive properties of the human brain and human behaviour are the result of something other than what we have less of than corn. There is now strong evidence that the complexity of higher organisms correlates with the relative amount of noncoding ribonucleic acid (RNA) rather than the number of protein-coding genes (Barrett et al., 2012). Noncoding RNA is part of a vast regulatory system and accumulated evidence indicates that the complexity of higher organisms arises from an increase in the number and complexity of regulatory pathways and that variation within these pathways produces phenotypic variation between both individuals and species.

These regulatory pathways do not 'regulate' deoxyribonucleic acid (DNA) in as much as DNA has no independent activity to be regulated. These pathways are part of the system whereby the cell utilises the DNA molecule, and a good deal else, in the process of phenotype creation. This shift from an exclusive focus on DNA sequence to the cellular processes that utilise the DNA sequence represents the 'post' in postgenomics. These cellular process - epigenetic regulation of DNA transcribability, noncoding RNAs, alternative splicing (AS) and post-translational modifications (PTMs) - appear to be particularly prevalent in, and of distinctive importance to, the human brain. These processes can be highly environmentally responsive and appear to play a key role in enabling the plasticity of the human brain and behaviour. This environmental responsiveness points to the critical importance of 'developmental windows', both pre- and postnatal, and the developmental process more generally, as an important force in behavioural developmental outcomes, both healthy and abnormal. It also serves to blur the distinction between 'nature' and 'nurture' or 'genes' and 'environment' that has been a fixation of behavioural genetics for so long. Because the scope, relevance and mechanisms of several of these processes are just beginning to be 
uncovered, the study of their influence on behaviour is still a fledgling science.

\section{Epigenetics}

Epigenetics is the study of mitotically and/or meiotically heritable changes in gene function that occur without changes to the DNA sequence. Cellular differentiation is an epigenetic process. The majority of human cells contain the same genome. Epigenetic programming during embryo genesis results in a variety of highly differentiated cells exhibiting distinct profiles of gene expression, cellular morphology and function (Goldberg et al., 2007). This epigenetic programming is heritable and stable, that is, differentiated cells only give rise to cells of the same kind through mitosis. However, epigenetic programming can also be variable and reversible. The inputs for epigenetic programming can come from environments ranging from the cellular to the maternal to the more broadly ecological. Although a variety of processes could potentially be described as epigenetic, the term is commonly limited to epigenetic changes involving alterations to chromatin - histone modification and DNA methylation - and the activity of noncoding RNAs, including micro RNA (miRNA). Although often investigated independently, these three epigenetic processes interact with one another in complex ways. See also: DNA Methylation and Histone Acetylation

Epigenetic processes are key to every aspect of development from the transition of an ovum to a state of totipotency, involving the erasure (albeit incomplete) of the preexisting epigenetic marks of gametic DNA, to cellular differentiation, involving the spatiotemporally coordinated establishment of differential epigenetic marks (Charney, 2013). They are critical for the differentiation of neural precursor cells (NPCs) from embryonic stem cells, the differentiation of distinct classes of neurons from NPCs, synaptogenesis and brain morphology, and appear to play key roles in synaptic plasticity, learning and memory.

\section{Prenatal environment}

Genomic imprinting is an epigenetic phenomenon in which genes are expressed in a monoallelic and parent of originspecific manner either from the paternal or maternal allele, whereas the inactive allele is repressed through the insertion of methylation marks at specific DNA sequences. To date, approximately 100 imprinted genes have been described in mammals (for a complete list, see http:// www.har.mrc.ac.uk/research/genomic_imprinting/ or http://igc.otago.ac.nz/home.html) and at least 60 in humans, and there are likely several hundred (Kelsey and Bartolomei, 2012). In addition to allele-specific methylation, imprinting is also associated with histone modifications and noncoding RNA including miRNA. See also: Genetic Conflict and Imprinting
The mammalian placenta is linked to the evolution of genomic imprinting and studies have shown that a number of imprinted genes are expressed in both the developing hypothalamus and placenta (Davies et al., 2008). In mammals, neuroendocrine regulation of maternal care is dependent on oestrogen-oxytocin interactions. The hormones produced by the placenta act on the maternal hypothalamus to silence sexual behaviour and reproduction, increase maternal feeding and prime the brain for postpartum maternal care by activating gene expression for synthesis of oxytocin and its receptors. Mice null for the maternally imprinted/paternally expressed genes, Peg1 and $P e g 3$, both of which are strongly expressed in the brain, exhibit a reduction in oxytocin neurons and reductions in quality of maternal care (Champagne et al., 2009). When Peg3 transcription is inactivated in the hypothalamus of the pregnant mother carrying wild-type offspring, the functional phenotypic outcomes are very similar to those that occur when the same gene is inactivated selectively in the developing placenta and fetal hypothalamus in a wildtype mother (Keverne, 2009).

The methylation of somatic imprinted genes is actively maintained throughout embryogenesis and the failure to maintain methylation marks can result in epigenetic disorders. Abnormal imprinting (epimutations) can lead to neurodevelopmental abnormalities associated with cognitive deficits such as Rett syndrome, Angelman syndrome or Prader-Willi syndrome (Chiba et al., 2013). An increased incidence of Angelman syndrome as well as the imprinting disorder Beckwith-Wiedemann syndrome has been associated with conception via in vitro fertilisation (IVF) (Hiura et al., 2012). Although this association remains controversial, numerous animal studies have indicated an association between IVF and imprinting abnormalities some involving the same genes as in human imprinting disorders - suggesting that the environmental perturbations associated with IVF may disrupt epigenetic processes in the zygote associated with genomic imprinting (Hori et al., 2010). See also: Genetic Imprinting in the PraderWilli and Angelman Syndromes

The developmental origins of disease or fetal programming model predicts that early exposures to threat or adverse conditions have lifelong consequences that result in poor health outcomes (Barker, 1998). Programming is a process by which a stimulus or exposure during a critical developmental period has a long-lasting or permanent influence on the brain, behaviour and risk for disease. Studies indicate that fetal exposure to elevated gestational stress and stress hormones can have significant and largely negative consequences for fetal, infant and child neurological development, resulting in delayed fetal maturation and impaired cognitive performance during infancy and decreased brain volume in areas associated with learning and memory in children, and is associated with behavioural and emotional disturbances during infancy and childhood (Sandman et al., 2011).

Altered miRNA expression may prime neuroplasticity and physiological processes in response to the early 
environment and may be a critical component in mediating the effects of prenatal stress on offspring development (Cohen et al., 2011). In rodents, prenatal stress modifies the expression of genes central to brain development and plasticity. In a recent study, analysis of new born brain global gene expression found 39 genes downregulated and 47 upregulated by prenatal stress, as well as 336 differentially expressed miRNAs including 131 downregulated and 205 upregulated (Zucchi et al., 2013; see Figure 1). The putative gene targets for these miRNAs included genes related to miRNA biogenesis, apoptosis, brain pathologies, neurotransmission, neurodevelopment, hormonal regulation, neurotrophic factors, brain angiogenesis, cell signalling, stress response and metabolism. Prenatal stress upregulated miR-103, which is involved in brain pathologies; downregulated miR-145, a marker of multiple sclerosis in humans; and upregulated miR-219, which targets the gene Dazap1. Both miR-219 and Dazapl are putative markers of schizophrenia and bipolar affective disorder in humans. miRNA expression appears to be altered in many common psychiatric and neurological disorders, such as bipolar disorder, schizophrenia, autism and depression conditions that share a suspected aetiology that includes both the influence of adverse perinatal origins as well as a transcriptome component (Petronis, 2010). See also: MicroRNAs in Animal Development

Depression during pregnancy is associated with increased maternal cortisol level and reduced gestational length and thus shares some common features with stress exposure during pregnancy (Gudsnuk and Champagne, 2011). Glucocorticoid receptor (GR) is a transcription factor involved in the regulation of the hypothalamicpituitary-adrenal (HPA) stress response through a negative feedback loop, with higher levels of GR associated with lower levels of stress responsivity and lower GR levels

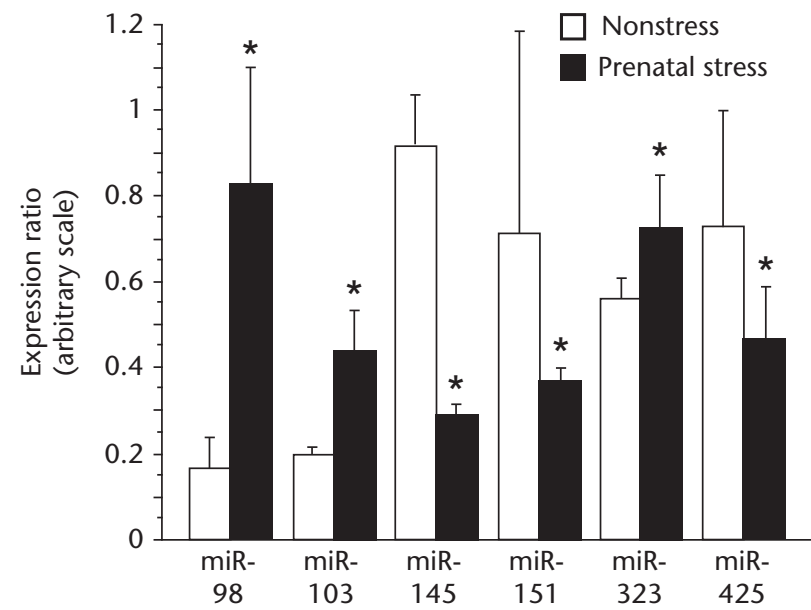

Figure 1 Prenatal stress modulates the brain miRNA expression in male newborn offspring. Expression ratio group averages of miRNAs from brains of newborns born to prenatally stressed dams. All data are presented as mean 6 SEM. Adapted and reprinted with permission from Zucchi et al. (2013, p. 4) and Nijholt (2004, p. 177). (C) PLoS. with higher stress responsivity. Analysis of cord blood samples from infants born to mothers with elevated ratings of depression (using the Hamilton Depression Scale) during the third trimester of pregnancy indicated elevated levels of DNA methylation within the GR promoter region (Oberlander et al., 2008). The degree of DNA methylation within the neonatal GR promoter was found to predict increased salivary cortisol levels in infants at 3 months of age.

\section{Postnatal environment}

The early rearing environment exerts a profound influence on developmental outcomes, both positive and negative. Animal studies have shown that differences in level of maternal care (as measured by licking and grooming (LG) behaviour), influence the stress response of offspring, with cross-fostered pups raised by high-LG dams exhibiting lower levels of stress as adults, whereas pups raised by low-LG dams exhibit higher levels of stress as adults (Szyf, 2009, 2013). As adults, the offspring of high LG mothers show decreased methylation of the binding site for the transcription factor nerve growth factor-inducible A (NGFIA) that lies within the exon $1_{7}$ promoter of $\mathrm{N1r3Cl}$ (the gene that encodes the GR in the hippocampus); this results in increased NGFIA binding to this promoter, increased hippocampal GR expression and more modest HPA responses to stress (Weaver et al., 2004; see Figure 2). In humans, child abuse is associated with increased methylation of the exon $1_{\mathrm{F}} G R$ gene promoter (the homologue of exon $1_{7}$ in rats) in the hippocampus (McGowan et al., 2009). These findings suggest that the effects of parental care may be mediated through a similar epigenetic mechanism in humans (Hackman et al., 2010).

As adults, cross-fostered female offspring of high- and low-LG mothers exhibit rearing behaviours that tend to match that of their rearing mother. In mammals, neuroendocrine regulation of maternal care is dependent on oestrogen-oxytocin interactions involving hypothalamic oestrogen receptors. In the rat, central oxytocin receptor levels are functionally linked to behavioural differences in maternal care. Dams who display high levels of maternal LG behaviour exhibit elevated expression of the oestrogen receptor $\alpha(E R \alpha)$ in a region of the hypothalamus known as the medial preoptic area, whereas low-LG dams exhibit decreased $E R \alpha$ expression. These differences in turn are associated with differences in DNA methylation: High-LG behaviour is associated with lower levels of methylation of the $\mathrm{ER} \alpha$ gene, which is associated with higher levels of $\mathrm{ER} \alpha$ transcription; low-LG behaviour is associated with higher levels of ER $\alpha$ gene methylation and lower levels of gene transcription (Cameron et al., 2008). Female offspring of low-LG mothers also exhibit increased sexual receptivity; increased levels of plasma luteinizing hormone, which regulates a number of aspects of the female menstrual cycle and accelerated puberty compared to offspring of high-LG mothers (Sakhai et al., 2011). There is evidence that human 
Tactile stimulation

(maternal licking and grooming)
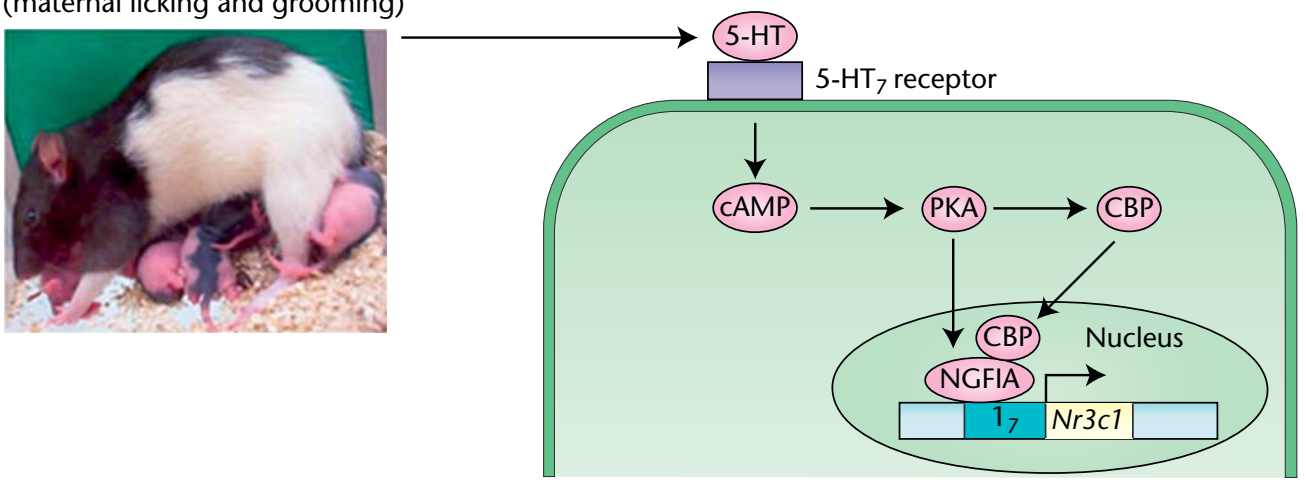

(a)

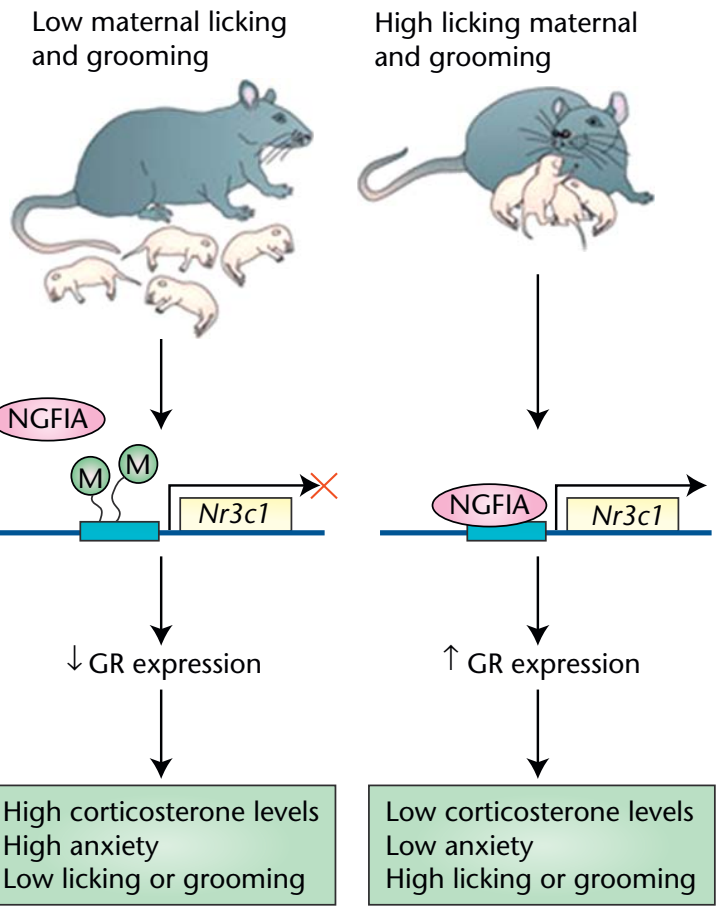

(b)

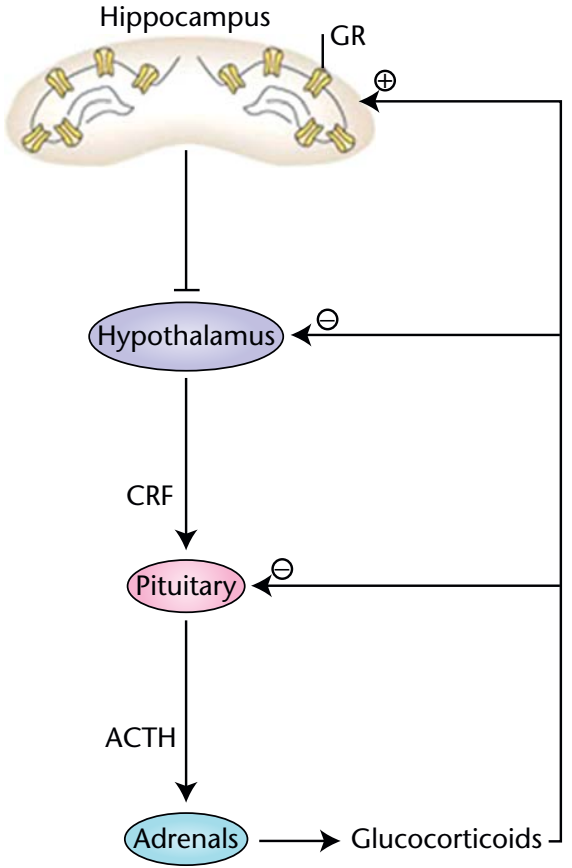

(c)

Figure 2 Parental regulation of the hypothalamic-pituitary-adrenal axis. (a) The current working model for the effect of maternal care (specifically, of licking and grooming pups) on the epigenetic regulation of the expression of $\mathrm{Nr} 3 \mathrm{c} 1$, the gene that encodes the GR. Licking and grooming of pups activates thyroid hormone-dependent increases in hippocampal serotonin (5-hydroxytryptamine or 5-HT) levels and 5-HT binding to the 5-HT7 receptor. Activation of the 5-HT7 receptor leads to the activation of a cyclic AMP-protein kinase A (PKA) cascade that induces the expression of the transcription factor nerve growth factor-inducible A (NGFiA) and cyclic AMP response element-binding (cReB) protein (cBP) expression and their association with the neuron-specific exon 17 GR gene promoter. (b) In neonates, high levels of licking increases NGFiA and cBP association with the exon 17 promoter by triggering demethylation of a dinucleotide sequence (cpG) that is located within the NGFiA binding region of the exon. This subsequently increases the ability of NGFiA to activate GR gene expression. M, methylation. (c) A schematic of the hypothalamic-pituitary-adrenal axis, the pivot of which are the corticotropinreleasing factor (CRF) neurons of the paraventricular nucleus of the hypothalamus. CRF is released into the portal system of the anterior pituitary, stimulating the synthesis and release of adrenocorticotropin (AcTH), which then stimulates adrenal glucocorticoid release. Glucocorticoids act on GRs in multiple brain regions, including the hippocampus, to inhibit the synthesis and release of cRF (i.e. glucocorticoid negative feedback takes place). The adult offspring of mothers that exhibit high licking and grooming, by comparison to those of low licking and grooming dams, show increased GR expression, enhanced negative-feedback sensitivity to glucocorticoids, reduced cRF expression in the hypothalamus and more modest pituitary-adrenal responses to stress. Adapted and reprinted with permission from Hackman et al. (2010, p. 656). @ Nature Publishing Group.

reproductive strategies in adverse environments exhibit a similar dynamic. Studies have shown that stressful early life conditions, including family relations, are associated with earlier age at menarche, and earlier age at menarche is associated with earlier onset of sexual behaviour and reproduction (De Genna et al., 2011). 


\section{Maternal Effects and Phenotypic Plasticity}

These are examples of maternal effects - maternal phenotype influencing the phenotype of offspring by other means than through the inheritance of maternal genes - a phenomenon found in almost all animal species; as well as adaptive phenotypic plasticity, the ability of an organism to change phenotype in response to the demands of its environment (Maestripieri and Mateo, 2009). Offspring behavioural plasticity enables the mother to adjust the phenotype of offspring in response to the environment she inhabits and, in doing so, in effect transmit to them information about the environment they will inhabit.

Adversity during perinatal development can forecast an increased level of demand in the environment the offspring will occupy. Under such conditions, the animal's best interest is to enhance its behavioural (e.g. vigilance and fearfulness) and endocrine (HPA and metabolic) responsiveness to stress (Champagne, 2010). These responses promote detection of potential threat, avoidance learning and metabolic/cardiovascular responses that are essential under the increased demands of the stressor. With regard to maternal rearing behaviour, under high-risk conditions, when the probability of extended periods of growth and survival are low, the optimal strategy is to maximise the number of offsprings through accelerated mating, increasing the chances that at least some offspring will survive to reproductive maturity. In contrast, more favourable environmental conditions favour greater investment in individual offspring at the cost of mating, since offspring quality predicts successful competition for available resources and reproductive fitness. Of course, a high degree of phenotypic plasticity may also entail that sometimes maternal effects can be maladaptive (as in the association between child abuse and mental illness). See also: Epigenetics: Influence on Behavioral Disorders

\section{AS and PTMs}

\section{AS}

Given that there are estimated to be more than 100000 proteins in the human body - and the number may be significantly higher - yet approximately 20000 genes in the human genome, necessitates a rethinking of the assumption that each gene contains the 'instructions' for making just one protein. AS allows a single gene to produce multiple transcripts and, consequently, multiple proteins, by utilising different splice sites. In gene transcription, exons and introns are first copied to create premessenger RNA (pre-mRNA). AS occurs when pre-mRNA exons are combined in various ways to form different mRNAs and proteins, called isoforms (Nilsen and Graveley, 2010). AS is estimated to occur in $95 \%$ of multiexonic human genes, and can result in numerous proteins being synthesised from the same gene (e.g. the neurexin proteins are encoded by three genes, but it is estimated that more than 1000 neurexin isoforms are produced by extensive AS). Isoforms of the same gene can exert radically different and even opposed physiological effects, and AS can also affect downstream regulatory processes and thus direct additional levels of post-transcriptional gene regulation.

Splicing alterations may involve changes in members of the serine-arginine (SR) rich proteins, implicated in AS and splice site selection (Tacke and Manley, 1999). Isoforms can also be produced by alternative promoter use, a widespread phenomenon in humans that can alter expression of the associated gene at both the mRNA and protein level. Alternative promoter use is an important mechanism in the cell- or developmental-specific expression of many genes, and global analysis of mammalian promoters show that alternative promoters are overrepresented among genes involved in transcriptional regulation and development (Baek et al., 2007). The collective mRNA and protein output that results from AS and alternative promoter usage constitutes the 'splicesome'. See also: Protein Isoforms and Isozymes

Distinct AS patterns are observed in different cellular environments. Such patterns can be tissue-specific (e.g. a sequence corresponding to a particular exon is included in muscle but not brain cells) or dictated by developmental or differentiation-specific cues (Pal et al., 2011). Neuronal isoform expression can be highly specific with individual neurons in a circuit or ganglion expressing different spliced isoforms of important transcripts. Compared to other human organs, the brain is known to express the largest number of distinct alternatively spliced transcripts, both diversifying the brain proteome and increasing neuronal gene regulation complexity (Norris and Calarco, 2012). Changes in AS have been shown to have important roles in many human neurological developmental processes, including neuronal cell migration, synapse formation and establishing neurotransmitter receptors (Grabowski, 2011). Failure or dysregulation of AS can cause abnormal neural development and neuropathology and has been associated with a number of neurodegenerative and psychiatric disorders (Mills and Janitz, 2012). See also: Alternative Splicing and Human Disease

Some AS patterns have been shown to be modulated in response to external stimuli, such as depolarisation of neurons or activation of signal transduction cascades ( $\mathrm{Li}$ et al., 2007), and AS patterns of numerous neuronally expressed genes have been found to be modified following stress insults. Acetylcholinesterase (AChE) is responsible for the rapid hydrolysis of acetylcholine and termination of neurotransmission in cholinergic synapses in the brain and occurs in at least three isoforms: 'synaptic' AChE-S (also known as AChE-T); AChE-R and the erythrocytic AChE$\mathrm{E}$ (see Figure 3). Several lines of evidence suggest that AChE-S and AChE-R have distinct, and sometimes inverse, roles in the normal brain (Meshorer and Soreq, 2006). In the normal human brain, much more AChE-S ('S' for 'synatpic') than AChE-R ('R' for 'read-through') mRNA is produced, but following stress, AChE-S production is downregulated and AChE-R upregulated (see 


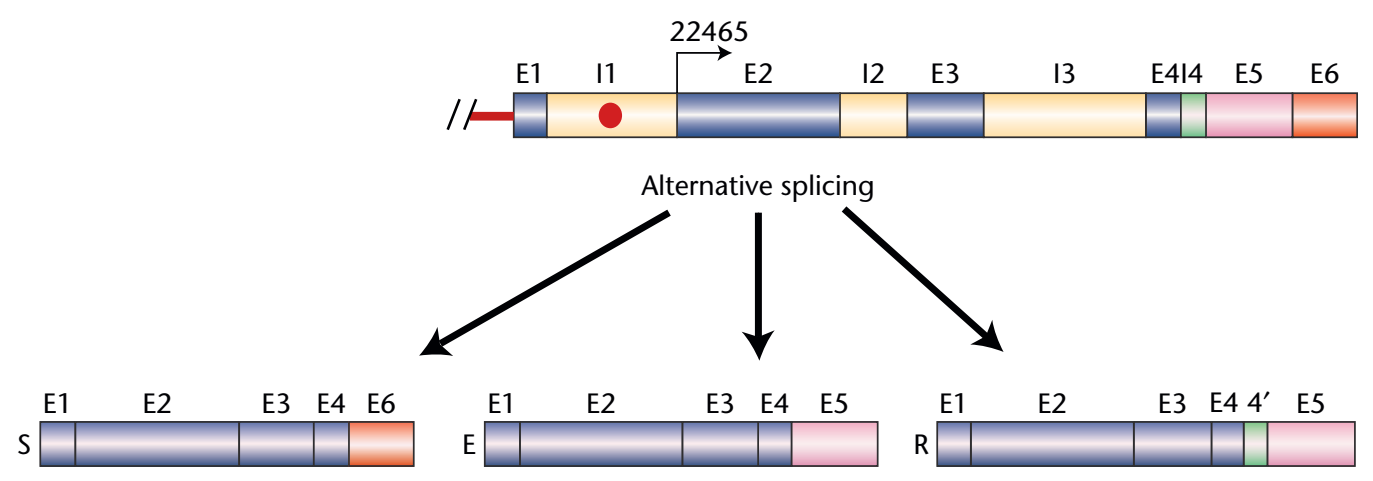

Figure 3 The human ACHE gene and its alternative messenger RNAs. The core of human AChE is encoded by three exons and parts of additional regions encode the variant-specific terminal sequences. Transcription begins at E1, and E2 encodes a leader sequence that does not appear in any mature protein. In addition to a proximal promoter (red line adjacent to E1), a distal enhancer region (more distal red line) is rich in potential regulatory sequences, some of which are shown as wedges. Intron 1 (I1) contains an enhancer sequence indicated by a red dot. Nucleotide numbers are those of GeneBank cosmid AF002993. Normally, much more AChE-S than AChE-R mRNA is produced, but under stress or inhibition of AChE, AS produces much more of the AChE-R mRNA. Modified and reprinted with permission from Soreq and Seidman (2001, p. 299). ๑ Nature Publishing Group.

Figure 4). In poststress processes in healthy volunteers, the blood levels of soluble AChE-R monomers were found to be inversely proportional to trait anxiety - the tendency to become anxious that reflects a combination of demographic determinants (e.g. age, ethnic origin, gender and body-mass index) and experience-derived elements (Sklan et al., 2004). This suggests that the capacity to overproduce AChE-R in reaction to various stressors may be an important determinant of the ability to cope with anxious stimuli.

\section{PTMs}

PTMs alter the properties of a protein by the addition of a modifying chemical group or another protein to one or more of its amino acid residues (Deribe et al., 2010). Histone modification is itself a PTM: 'post-translated' histone proteins are subject to a wide variety of chemical modifications that alter their behaviour. In fact, most proteins, once translated, are subject to some form of PTM. More than 400 discrete types of modifications can occur, and to date, more than 90000 individual PTMs have been identified through biochemical and biophysical analysis (Lorthrop et al., 2013). The extensive array of reversible PTMs creates diversity in signalling and is particularly suitable for relaying rapid messages in the cell. Some PTMs are stable and are important for maturation and proper folding of newly synthesised proteins, while others are more transient and have essential roles in intracellular signalling. A protein can be modified by more than one type of PTM and modified multiple times by the same PTM at different residues.

Consider, as just one example, the PTM ubiquitination, which involves the covalent attachment of the 76 amino acid protein ubiquitin to a substrate protein. Ubiquitination regulates a myriad of cellular processes, including cell division, differentiation, signal transduction, protein trafficking and protein quality control (Haas and
Broadie, 2008). One of the effects of ubiquitination is to mark a protein for proteasomal degradation as part of an ubiquitin proteasome system (UPS). Protein degradation is now recognised alongside protein translation as a primary means for regulating the abundance and trafficking of proteins critical for neurotransmission. The UPS functions locally at neuronal synapses, and its various roles include feedback control of transcription, coordination of synapse development versus elimination, local maintenance of neurotransmission strength and mediating activity-dependent modulation of pre- and postsynaptic function (Yi and Ehlers, 2007). Aggregation of proteins is a major feature of several neurodegenerative disorders and several lines of evidence suggest the potential involvement of the UPS in Alzheimer disease, Huntington's disease and Parkinson disease (Vidal, 2010).

\section{Inheritance}

DNA is not the sole biological agent of inheritance. In addition to the epigenetic marks associated with imprinting, there is growing evidence that epigenetic changes can be inherited via the germline as well as somatically, resulting in the intergenerational nongenomic inheritance of epigenetic states. Maternal oocytic mRNAs and miRNAs play critical roles in early embryonic development and before the activation of the embryonic genome, the translation of maternal mRNAs (by maternal organelles) regulates every aspect of zygotic development. Mitochondria and mitochondrial DNA, which play key roles in neuronal development and function, are maternally also inherited, as are nucleoli, and an assortment of proteins, macromolecules and organelles (Charney, 2012). Sperm transmit miRNAs that play critical roles in early development (they are necessary for the first cleavage in mice) other noncoding RNAs believed to modify important postfertilisation events, and centrosomes (Kumar et al., 2013). What is 

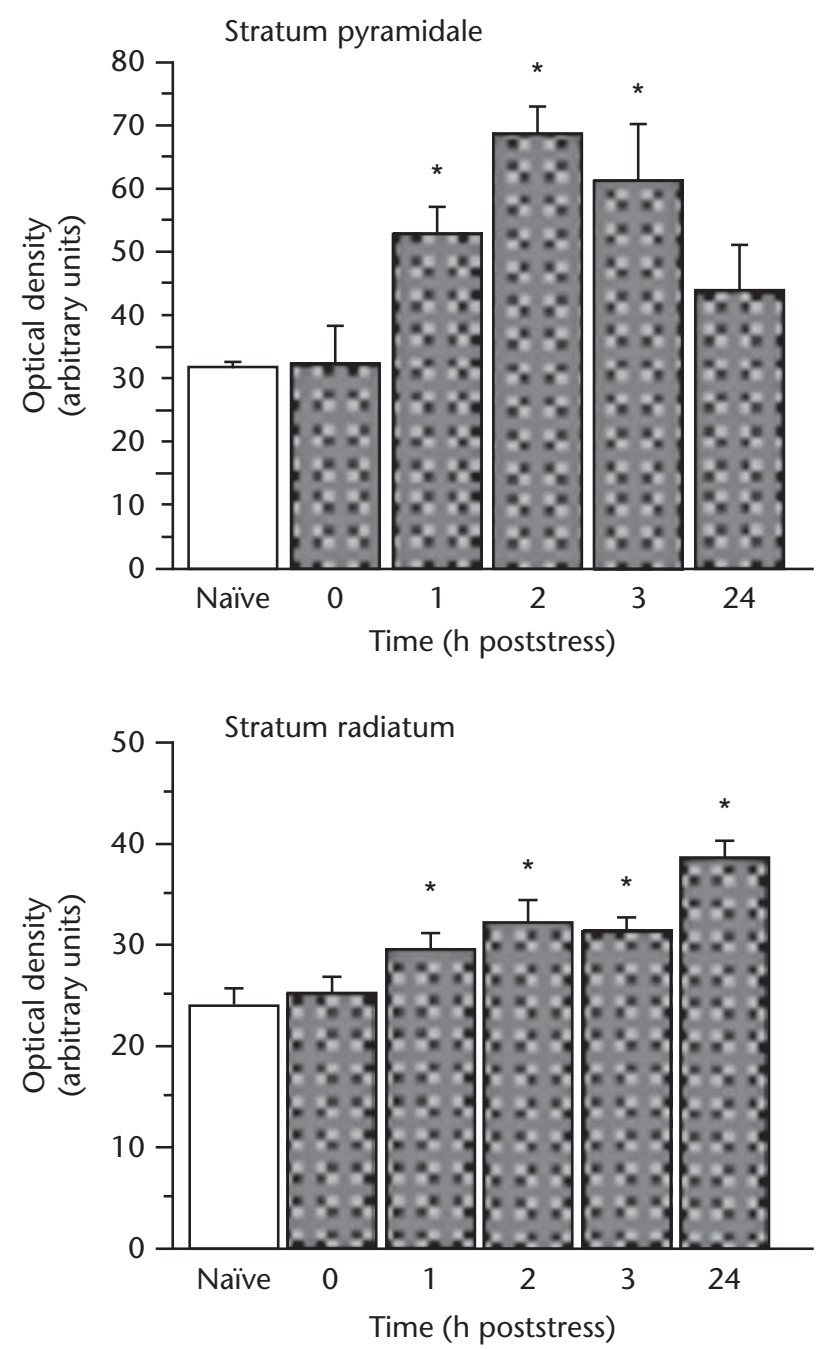

Figure 4 Acute immobilisation stress induces AChE-R upregulation in hippocampal CA1 neurons. Bars represent densitometric analysis (mean 7SEM; both hippocampi of $\mathrm{n} 1 / 45$ per group) of AChE-R-positive cells in the stratum pyramidale and stratum radiatum. Statistically significant differences: ${ }^{*} p<.05$ versus naïves. Adapted and reprinted with permission from Nijholt (2004, p. 177). (c) Nature Publishing Group.

important to note is that all of these constitute non-DNA based, non-Mendelian forms of inheritance. Their phenotypic effects cannot be quantified by any standard measures of heritability.

\section{Conclusion}

From a postgenomics perspective, the operating assumption should be that when it comes to human behaviour, and particularly complex behaviour associated with the healthy brain, genotype alone will neither explain nor predict phenotype (or phenotypic variance), whether we are talking about one polymorphism or a thousand (where each polymorphism is presumed to be of tiny effect). The proliferation of '-omes' and 'omics' - the transcriptome, the proteome, the splicesome, the metabalome - represents a postgenomic shift in at least two ways: First, the level of analysis has shifted from the genome per se to the cell (as the smallest unit of analysis) and second, the various '-omes' are conceived of as dynamic, interacting systems, rather than the outputs of the unvarying template of heredity (i.e. DNA). Furthermore, in the brain, epigenomes, transcriptomes, proteomes and splicesomes can vary from neuron to neuron, and there is growing evidence that neuronal DNA itself varies as a result of retrotransposons, copy number variations and aneuploidy - all resulting in a brain characterised by somatic mosaicism. See also: Somatic Genome Variations

The incorporation of newly discovered heritable agents and assorted omics is still likely to yield insufficient understanding, however, without a thorough consideration of possible environmental factors as contributing agents. From the postgenomics perspective, the environment is as much a carrier of information as the DNA sequence, both for shaping phenotype and as a source of information in trying to predict phenotype. An awareness of developmental biology and the concept of phenotypic plasticity need to inform any search for predictors of behaviour, and known domains and mechanisms of plasticity should be a part of every investigation. This begins, naturally, with the fetal environment. Hence, researchers need to examine not just the parents' genomes, but the mother's behaviour during pregnancy and birth - her nutrition, stress levels, lifestyle, mode of interaction with offspring, levels of maternal care, breast-feeding behaviour and so on.

Finally, one of the key insights to emerge from the resurrection of developmental biology as a central focus in evolutionary theory is that development occurs not simply in the mother's womb, but in an ecological niche (West and King, 1987). For better or worse, the ecological environment of the mother impacts the perinatal environment. Hence, we also need to incorporate into an expanded synthesis an understanding of the broader ecological factors: socioeconomic status, environmental toxins, health care provision, and even weather and light and day cycles, all of which can also shape phenotype. In contemporary evolutionary theory, an ambitious synthesis of all these forces has been fashioned into an area of research dubbed ecological developmental biology. A parallel approach in the behavioural sciences may yield valuable insights into human behaviour in the postgenomics era. See also: Ecological Development Biology

\section{References}

Baek DC, Davis B, Ewing B, Gordon D and Green P (2007) Characterization and predictive discovery of evolutionarily conserved mammalian alternative promoters. Genome Research 17(2): 145-155.

Barker DJP (1998) Mothers, Babies and Health in Later Life. Edinburgh: Harcourt Brace \& Co., Ltd. 
Barrett LW, Fletcher S and Wilton SD (2012) Regulation of eukaryotic gene expression by the untranslated gene regions and other non-coding elements. Cellular and Molecular Life Sciences 69(21): 3613-3634.

Cameron NM, Shahrokh D, Del Corpo A et al. (2008) Epigenetic programming of phenotypic variations in reproductive strategies in the rat through maternal care. Journal of Neuroendrochronology 20: 795-801.

Champagne FA (2010) Early adversity and developmental outcomes. Perspectives on Psychological Science 5(5): 564 574.

Champagne FA, Curley JP, Swaney WT, Hasen NS and Keverne EB (2009) Paternal influence on female behavior: the role of Peg3 in exploration, olfaction, and neuroendocrine regulation of maternal behavior of female mice. Behavioral Neuroscience 123(3): 469-480.

Charney E (2012) Behavior genetics and postgenomics. Behavioral and Brain Sciences 35(5): 331-358.

Charney E (2013) Cytoplasmic inheritance redux. Advances in Child Development and Behavior 44: 225-255.

Chiba H, Hiura H, Okae H et al. (2013) DNA methylation errors in imprinting disorders and assisted reproductive technologies. Pediatrics International 55(5): 542-549.

Cohen JE, Lee PR, Chen S, Li W and Fields RD (2011) MicroRNA regulation of homeostatic synaptic plasticity. Proceedings of the National Academy of Sciences of the USA 108(28): 1165011655.

Davies W, Isles AR, Humbly T and Wilkinson LS (2008) What are imprinted genes doing in the brain? Advances in Experimental Medical Biology 626: 62-70.

De Genna NM, Larkby C and Cornelius MD (2011) Pubertal timing and early sexual intercourse in the offspring of teenage mothers. Journal of Youth and Adolescence 40(10): 13151328.

Deribe YL, Pawson T and Dikic I (2010) Post-translational modifications in signal integration. Nature Structural \& Molecular Biology 17(6): 666-672.

Goldberg AD, Allis CD and Bernstein E (2007) Epigenetics: a landscape takes shape. Cell 128(4): 635-638.

Grabowski P (2011) Alternative splicing takes shape during neuronal development. Current Opinion in Genetics \& Development 21: 388-394.

Gudsnuk KM and Champagne FA (2011) Epigenetic effects of early developmental experiences. Clinics in Perinatology 38(4): $703-717$.

Haas KF and Broadie K (2008) Roles of ubiquitination at the synapse. Biochimica et Biophysica Acta 1779(8): 495-506.

Hackman DA, Farah MJ and Meaney MJ (2010) Socioeconomic status and the brain: mechanistic insights from human and animal research. Nature Reviews Neuroscience 11(9): 651-659.

Hiura H, Hiroaki O, Naoko M et al. (2012) Characterization of DNA methylation errors in patients with imprinting disorders conceived by assisted reproduction technologies. Human Reproduction 27(8): 2541-2548.

Hori N, Nagai M, Hirayama M et al. (2010) Aberrant CpG methylation of the imprinting control region KvDMR1 detected in assisted reproductive technology-produced calves and pathogenesis of large offspring syndrome. Animal Reproduction Science 122(3-4): 303-312.
Kelsey G and Bartolomei MS (2012) Imprinted genes ... and the number is? PLoS Genetics 8(3): e1002601.

Keverne B (2009) Monoallelic gene expression and mammalian evolution. BioEssays 31(12): 1318-1326.

Kumar M, Kumar K, Jain S, Hassan T and Dada R (2013) Novel insights into the genetic and epigenetic paternal contribution to the human embryo. Clinics 68(suppl. 1): 5-14.

Li Q, Lee J-A and Black DL (2007) Neuronal regulation of alternative pre-mRNA splicing. Nature Reviews Neuroscience 8(11): 819-831.

Lorthrop AP, Torres MP and Fuchs SM (2013) Deciphering posttranslational modification codes. FEBS Letters 587(8): 12471257

Maestripieri D and Mateo JM (2009) Maternal Effects in Mammals. Chicago: University of Chicago Press.

McGowan PO, Sasaki A, D'Alessio AC et al. (2009) Epigenetic regulation of the glucocorticoid receptor in human brain associates with childhood abuse. Nature Neuroscience 12(3): 342-348.

Meshorer E and Soreq H (2006) Virtues and woes of AChE alternative splicing in stress-related neuropathologies. Trends in Neuroscience 29(4): 216-224.

Mills JD and Janitz M (2012) Alternative splicing of mRNA in the molecular pathology of neurodegenerative diseases. Neurobiology of Aging 33(5): 1012e11-1012e24.

Nijholt I, Farchi N, Kye M et al. (2004) Stress-induced alternative splicing of acetylcholinesterase results in enhanced fear memory and long-term potentiation. Molecular Psychiatry 9(2): 174-183.

Nilsen TW and Graveley BR (2010) Expansion of the eukaryotic proteome by alternative splicing. Nature 463(7280): 457-463.

Norris AD and Calarco JA (2012) Emerging roles of alternative pre-mRNA splicing regulation in neuronal development and function. Frontiers in Neuroscience 6: 122.

Oberlander TF, Weinberg J, Papsdorf M et al. (2008) Prenatal exposure to maternal depression, neonatal methylation of human glucocorticoid receptor gene (NR3C1) and infant cortisol stress responses. Epigenetics 3(2): 97-106.

Pal S, Gupta R, Kim H et al. (2011) Alternative transcription exceeds alternative splicing in generating the transcriptome diversity of cerebellar development. Genome Research 21(8): $1260-1272$.

Petronis A (2010) Epigenetics as a unifying principle in the aetiology of complex traits and diseases. Nature 465(7299): 721-727.

Sakhai SA, Kriegsfeld LJ and Francis DD (2011) Maternal programming of sexual attractivity in female Long Evans rats. Psychoneuroendocrinology 36(8): 1217-1225.

Sandman CA, Davis EP, Buss C and Glynn LM (2011) Exposure to prenatal psychobiological stress exerts programming influences on the mother and her fetus. Neuroendocrinology 15: 15.

Sklan EH, Lowenthal A, Korner M et al. (2004) Acetylcholinesterase/paraoxonase genotype and expression predict anxiety scores in health, risk factors, exercise training, and genetics study. Proceedings of the National Academy of Sciences of the USA 101(15): 5512-5517.

Soreq H and Seidman S (2001) Acetylcholinesterase-new roles for an old actor. Nature Reviews Neuroscience 2(4): 294-302. 
Szyf M (2009) The early life environment and the epigenome. Biochimica et Biophysica Acta 1790(9): 878-885.

Szyf M (2013) DNA methylation, behavior and early life adversity. Journal of Genetics and Genomics 40(7): 331-338.

Tacke R and Manley JL (1999) Determinants of SR protein specificity. Current Opinion in Cell Biology 11(3): 358-362.

Turkheimer E (2012) Genome wide association studies of behavior are social science. In: Plaisance KS and Reydon TAC (eds.) Philosophy of Behavioral Biology, pp. 43-64. Netherlands: Springer.

Vidal CJ (2010) Post-Translational Modifications in Health and Disease. New York: Springer.

Weaver IC, Cervoni N, Champagne FA et al. (2004) Epigenetic programming by maternal behavior. Nature Neuroscience 7(8): 847-854.

West MJ and King AP (1987) Settling nature and nurture into an ontogenetic niche. Developmental Psychobiology 20(5): 549562.

Yi JJ and Ehlers MD (2007) Emerging roles for ubiquitin and protein degradation in neuronal function. Pharmacological Reviews 59(1): 14-39.

Zucchi FCR, Yao Y, Ward ID et al. (2013) Maternal stress induces epigenetic signatures of psychiatric and neurological diseases in the offspring. PLoS One 8(2): e56967.

\section{Further Reading}

Gilbert SF (2012) Ecological developmental biology: environmental signals for normal animal development. Evolution \& Development 14(1): 20-28.

Gilbert SF and Epel D (2009) Ecological Developmental Biology: Integrating Epigenetics, Medicine, and Evolution. Sunderland, MA: Sinauer Associates.

Hood KE, Halpern CT, Greenberg G et al. (2010) The Handbook of Developmental Science, Behavior, and Genetics. Malden, MA: Blackwell Publishing Ltd.

Jablonka E and Lamb MJ (2006) Evolution in four Dimensions: Genetic, Epigenetic, Behavioral, and Symbolic Variation in the History of Life. Cambridge, Mass: MIT Press.

Lewontin RC (2000) The Triple Helix: Gene, Organism, and Environment. Cambridge, Mass: Harvard University Press.

Noble D (2010) Biophysics and systems biology. Philosophical Transactions of the Royal Society A: Mathematical, Physical and Engineering Sciences 368(1914): 1125-1139. 